\title{
MALADIES TRANSMISSIBLES ET ALTERATION DE LA BIODIVERSITE : QUELLES RELATIONS ?
}

\author{
COMMUNICABLE DISEASES AND DETERIORATION OF BIODIVERSITY: \\ WHAT ARE THE RELATIONSHIPS?
}

Par Emmanuelle ROBARDET ${ }^{1}$, Nathalie CHARBONNEL², Jean-François. GUÉGAN ${ }^{3}$, Elodie MONCHÂTRE-LEROY ${ }^{4}$, André PARODI ${ }^{5}$, Jean-Yves. GAUCHOT ${ }^{6}$, Hervé ZELLER ${ }^{7}$, Didier BOUSSARIE ${ }^{8} \mathcal{E}^{2}$ Marc ARTOIS $^{9}$

(Manuscrit accepté le 14 février 2021)

\begin{abstract}
RÉSUMÉ
Les auteurs réunis sous l'égide de l'Académie Vétérinaire de France ont étudié comment la littérature scientifique interroge le rapport entre l'émergence de zoonoses ou de nouvelles maladies infectieuses humaines et animales, d'une part et les pertes de biodiversité observées sous l'effet des pressions humaines d'autre part. L'examen de la littérature consultée décrit comment certains agents pathogènes peuvent passer du " réservoir " naturel sauvage à celui des animaux domestiques et aux populations humaines. L'exploitation et la destruction des milieux naturels comme les forêts primaires et les zones humides tropicales, ainsi que le rapprochement d'espèces consécutif, favorisent des contacts permettant le passage (direct ou via des réservoirs intermédiaires) d'agents pathogènes du réservoir sauvage aux humains, qui propagent ensuite ces agents sur la planète. II existe toutefois une importante variabilité des modalités permettant le passage, puis la propagation locale et planétaire des agents pathogènes issus de la biodiversité. Il est prématuré de tirer des conclusions générales sur les relations que cette revue scientifique a examinées. L'article suggère néanmoins que l'approche pluridisciplinaire doit progresser tant dans le domaine de la recherche que dans celui des pratiques et de l'enseignement. Cette pluridisciplinarité implique une étroite collaboration entre les médecines vétérinaires et humaines, l'écologie de la santé, d'autres disciplines biologiques et environnementales, et les sciences humaines.
\end{abstract}

Mots-clés : biodiversité, transmission, zoonose, maladie infectieuse émergente

\footnotetext{
(1) Laboratoire de la Rage et de la Faune Sauvage de Nancy, ANSES, Bâtiment H, Technopôle Agricole et Vétérinaire CS 4000954220 Malzéville, France. emmanuelle.robardet@anses.fr. Auteur correspondant

(2) CBGP, INRAE, CIRAD, IRD, Institut Agro, Université de Montpellier, Montpellier, France nathalie.Charbonnel@inrae.fr.

(3) ASTRE, INRAE, CIRAD, Université de Montpellier, Campus international de Baillarguet, 34398 Montpellier Cedex 5, France ; MNEGEC,

IRD, CNRS, Université de Montpellier, 911 avenue Agropolis, BP 64501, 34394 Montpellier cedex 5, France jean-francois.guegan@inrae.fr.

(4) Laboratoire de la Rage et de la Faune Sauvage de Nancy, ANSES, Bâtiment H, Technopôle Agricole et Vétérinaire CS 4000954220.

Malzéville, France. elodie. monchatre-leroy@anses.fr

(5) Président honoraire de l'Académie nationale de Médecine et de l'Académie vétérinaire de France, Directeur honoraire de l'Ecole nationale vétérinaire d'Alfort. andre-laurent.parodi@vet-alfort.fr.

(6) DMV, praticien, Clinique Vétérinaire, Terre de Fontenille 24260 Le Bugue, Fédération des Syndicats Vétérinaires de France Maison des Vétérinaires 10 place Léon Blum 75011 Paris JYGauchot@wanadoo.fr.

(7) Anciennement Centre européen de prévention et de contrôle des maladies (ECDC), Gustav III, Boulevard 40, 16973 Solna, Suède

herve.zeller@ecdc.europa.eu, et Institut Pasteur, Paris.

(8) Dr vét. Clinique vétérinaire Fismes 2 rue des Comtes Thibault 51170 FISMES didier.boussarie@wanadoo.fr.

(9) LISAE : Lorraine investigation santé animale et environnementale. 54200 Lagney lisae@orange.fr.
} 


\begin{abstract}
-ABSTRACT
The authors, under the aegis of the Veterinary Academy of France, studied how the scientific literature questions the relationship between the emergence of zoonosis or new human and animal infectious diseases and the loss of biodiversity observed under anthropogenic changes. This review describes how some pathogens can spill over from "wild reservoir " to domestic animal populations and human. The exploitation and destruction of natural environments such as primary forests and tropical wetlands, and closest proximity amongst species that is consecutive promote contacts that allow pathogens to circulate from the wild natural reservoirs to humans, who then spread these agents all over the world. However, there is a large array of modalities with regard to the transmission and further spread of pathogens from biodiversity. It is premature to draw general conclusions on these relationships from this review only. However, the study emphasizes the needs to strengthen a multidisciplinary approach in research as well as in practice and teaching. Such multi-disciplinary approach should involve close collaborations between veterinary and human medicines, ecology, other biological and environmental disciplines and the human sciences.

Key words : biodiversity, transmission, zoonosis, infectious emerging disease.
\end{abstract}

\section{INTRODUCTION}

Le recours aux vaccins et aux antimicrobiens a un temps laissé croire que l'humanité pourrait contrôler les maladies infectieuses et parasitaires. Dès les années 1980, l'irruption de maladies infectieuses inconnues au préalable, comme le SIDA (syndrome d'immunodéficience acquise) et la légionellose, a remis en cause cet optimisme et promu le concept de « maladies infectieuses émergentes (MIE) » et aussi « réémergentes » lorsque des maladies ayant précédemment disparu, ou régressé, ont réapparu. La notion d'agents infectieux émergents a fait l'objet d'une littérature grandissante dès la décennie 1990 (Figure 1), en particulier à la suite de la découverte du virus de l'immunodéficience humaine (VIH) responsable du SIDA en 1983. Par la suite, d'autres maladies émergentes ont aussi relevé l'attention de la communauté scientifique (Morse et al., 2012), avec notamment la Grippe A induite par le virus Influenza hautement pathogène H5N1 en 1997 puis en 2005 (Alexander, 2007), le virus Influenza H1N1 en 2009 (Nava et al., 2009), puis le syndrome respiratoire aigu sévère (SRAS) en 2003 lié au virus SRAS-CoV-1 (Berger et al., 2004), le syndrome respiratoire du Moyen-Orient (MERS) en 2012 dû au virus MERS-CoV (Abdallat et al., 2013) pour finir par la COVID-19 en 2020 et le virus SRAS-CoV-2 (Huang et al., 2020), en nous limitant, pour illustration, depuis le VIH, à des virus provoquant des maladies respiratoires susceptibles de déclencher des pandémies comme la COVID-19.

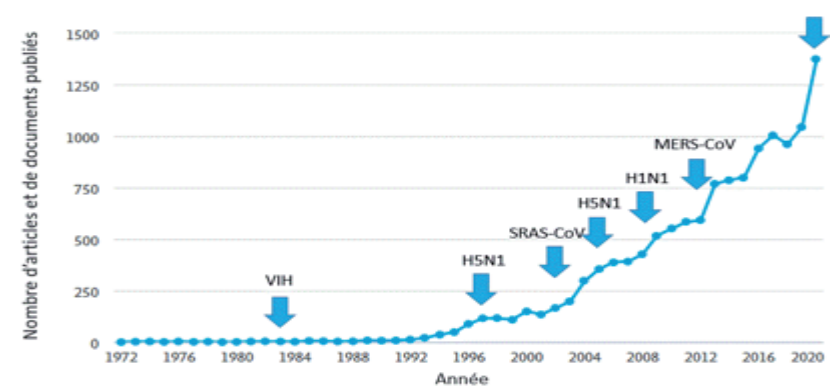

Figure 1 : Résultat de requête du nombre d'articles scientifiques de la base documentaire Scopus incluant les termes 'emerging infectious disease' au cours des cinquante dernières années, avec indication par des flèches de quelques exemples marquants de virus émergents.
Au cours des années 2000, des articles de synthèse (Taylor et al., 2001; Woolhouse et al., 2005; Jones et al., 2008) parus dans des revues scientifiques prestigieuses, révélaient au public les diverses catégories d'agents pathogènes qui étaient associées à ces émergences et montraient l'importance particulière des maladies humaines dues à des agents pathogènes entretenus et propagés par des animaux ou l'environnement. En complément de ces publications citées d'innombrables fois, d'autres études, en particulier celle de Cleaveland et al. (2001) ont montré que les maladies transmissibles des animaux domestiques répondaient à des critères analogues.

De ces études est ressorti l'argument, depuis largement vulgarisé, que la plupart des maladies infectieuses et parasitaires humaines (les chiffres varient selon les publications entre environ 1407 agents pathogènes humains répertoriés pour Woolhouse et al. (2005) et plus de 2100 pour Wardeh et al. (2015) et Murray et al. (2018) ont pour origine un agent pathogène " multi-hôtes " (ces hôtes sont selon les cas des animaux sauvages ou domestiques et des humains). Les émergences d'agents pathogènes sont actuellement représentées à plus de $62 \%$ par des agents d'origine animale (zoonoses) au sein desquels, ces 50-60 dernières années, $72 \%$ seraient issus de la faune sauvage (Jones et al., 2008; Murray et al., 2018). L'évolution des émergences d'agents pathogènes des 60 dernières années révèle une augmentation significative de leur occurrence (Jones et al., 2008; Morand \& Figuié, 2018) mettant en lumière des enjeux de mobilisation et de réorganisation de nos sociétés, tant d'un point de vue collectif qu'individuel (Ashford, 2003).

La problématique de l'origine faunistique des pathogènes émergents ressurgit régulièrement dans les médias en particulier à l'occasion d'alertes sanitaires et de menaces pandémiques. L'émergence du virus SARS-CoV-2 (Wu et al., 2020; Zhou et al., 2020) s'est donc sans surprise accompagnée de son lot d'interrogations médiatiques et de controverses, cette fois avec le pangolin comme emblème allégorique (Daszak et al., 2020; Salata et al., 2020; Xiao et al., 2020). Cependant, il a été depuis suggéré que la proximité génétique apparente entre les coronavirus de pangolins et le SARS-CoV-2 est probablement fortuite (Liu et al., 2020). 
La pandémie du SARS-CoV-2 a notamment été l'occasion de percevoir l'enjeu sociétal que constituent les épidémies. L'évaluation de la crise, la préparation des sociétés et les réponses apportées par les autorités sont autant d'éléments clés dans la conduite d'un programme de surveillance et de lutte sanitaire (Zylberman, 2013). Au-delà de la nécessaire réponse d'urgence faite à une crise sanitaire, il apparaît essentiel de pouvoir identifier et caractériser les agents pathogènes des maladies humaines ou animales et leur source, puis de comprendre leur propagation, afin de mieux anticiper le risque et les réponses à y apporter (Keck, 2020).

Le mémoire présenté ici porte essentiellement sur les relations écologiques susceptibles d'exister entre certains hôtes naturels sauvages d'agents pathogènes, et le passage de ces derniers à des espèces animales domestiques et/ou aux humains. Dans les paragraphes qui suivent, nous explorerons plus particulièrement le lien entre pertes d'origine anthropique de la biodiviersité et l'émergence de nouvelles maladies infectieuses. L'article abordera dans un premier temps les méthodes d'études, puis les maladies d'intérêt, et aussi les populations animales sources d'agents pathogènes, pour ensuite discuter de la sémantique des termes "santé " et "biodiversité ». Il abordera également la transmission et la propagation de ces agents de maladies, puis l'augmentation contemporaine de la fréquence des épidémies et enfin l'impact de la biodiversité sur la santé humaine et animale.

\section{METHODES D'ETUDES DES LIENS ENTRE ALTERATION DE LA BIODIVERSITE ET EMERGENCES DE CERTAINES MALADIES}

Pour l'essentiel, les études originales, notamment dans des programmes européens et américains, utilisent des approches descriptives et analytiques et plus rarement expérimentales sur lesquelles des synthèses sont fondées ; ces études portent sur des investigations microbiologiques (diagnostic et dépistage des agents pathogènes) et des enquêtes de terrain (approches épidémiologiques et écologiques des facteurs de risque d'infection ou de portage parasitaire (Abbate et al., 2020). Pour certaines de ces synthèses, des bases de données ont été compilées afin de relier statistiquement et par modélisation, d'une part les agents pathogènes découverts sur des animaux sauvages en liberté et, d'autre part les agents responsables de maladies humaines ou animales (animaux de production ou de compagnie) (Salkeld et al., 2013; Allen et al., 2017; Olival et al., 2017; Young et al., 2017). L'infection ou l'infestation des animaux sauvages n'entraîne pas toujours, voire rarement le développement d'une maladie cliniquement exprimée ou de lésions identifiables dans ces espèces ; leur importance voire leur présence peuvent donc être mésestimées. De ce fait, dans la plupart des études tout comme dans ce mémoire, ce ne sont pas les maladies des animaux sauvages qui retiennent prioritairement l'intérêt, mais principalement les maladies humaines ou des animaux domestiques, dont les agents sont entretenus et/ou transmis par les animaux sauvages.

Les synthèses conduites pour rechercher des liens entre altérations de la biodiversité et émergence de maladies infectieuses humaines ou d'espèces domestiques se fondent en grande partie sur des études réalisées à partir de travaux scientifiques originaux « les méta-analyses " qui répondent à des règles strictes pour être publiées ; ces synthèses reposent aussi sur des revues exhaustives de la bibliographie (Borenstein et al., 2009). Ces bases de données peuvent comprendre plusieurs centaines ou plusieurs milliers de liens « microbe / espèce hôte /maladie " permettant des traitements bio-statistiques appropriés et la révélation de dépendances (comme par exemple les proportions de maladies infectieuses humaines qui sont des zoonoses). Compte tenu de l'ampleur de l'éventail des triades microbe / hôte / maladie (Guégan et al., 2020), il est tentant de délimiter le champ des études (Exemple : virus / mammifères) au risque de créer des biais de sélection des sujets à prendre en considération. Les maladies humaines, par exemple, peuvent prendre le pas sur les maladies animales à impact économique. Les maladies à transmission vectorielle peuvent parfois mieux retenir l'attention que des maladies transmises différemment.

Récemment, ce type de travaux synthétiques a remis en exergue le rôle épidémiologique des chauves-souris connues pour héberger un grand nombre d'espèces virales sans en subir de conséquences cliniques (Calisher et al., 2006; Hayman, 2019). Ces espèces représentent-elles un réservoir privilégié d'agents pathogènes nouveaux pour l'humain, ou les ordres des Rongeurs et Chiroptères comptant le plus grand nombre d'espèces ont-ils été plus étudiés que tout autre groupe animal (Luis et al., 2013; Gibb et al., 2020) ?

Le rôle particulier de certains taxons comme source d'agents pathogènes humains reste donc débattu ; les fonctions et relations écologiques (prédation, commensalisme, domestication...) ou les proximités phylogénétiques (liées aux spécificités des récepteurs cellulaires permettant l'infection des cellules du nouvel hôte) contribuent à expliquer, en sus de la seule richesse spécifique des taxons, la possible contamination interspécifique entre populations d'animaux sauvages, domestiques et d'humains (Johnson et al., 2020). La pratique et le développement de l'élevage ont également favorisé la liaison épidémiologique plus fréquente avec des taxons tels que par exemple les Périssodactyles et Artiodactyles (autrefois "Ongulés ») (Woolhouse et al., 2005; Wells et al., 2020).

\section{LES MALADIES D'INTERET}

Cette revue porte principalement sur les maladies humaines, ainsi que certaines maladies des animaux domestiques de production et de compagnie, qui ont émergé ou réémergé en Europe ces dernières décennies. Toutefois, nous avons exclu de cette revue les maladies contagieuses qui actuellement sont entretenues et propagées uniquement au sein de l'espèce humaine, ainsi que des maladies des animaux domestiques qui ne sont pas transmises par des espèces animales sauvages porteuses des agents pathogènes.

Certaines maladies transmissibles humaines sont des zoonoses vraies (maladies naturellement transmises des animaux vertébrés aux humains, et réciproquement, comme la rage). Elles impliquent l'existence d'un réservoir animal indispensable pour entretenir l'agent pathogène et le communiquer aux humains ; sans cet animal, la transmission s'éteint. Certaines maladies transmissibles sont qualifiées de sapronoses (mot anglais, dont le 
meilleur équivalent en français serait « maladie tellurique ») car l'agent initial, le saprobionte, est naturellement entretenu dans un milieu ou un élément naturel (eau ou sol) et devient pathogène en contaminant une personne (c'est le cas des bactéries responsables de la légionellose).

Les maladies humaines (nous utiliserons par la suite la forme raccourcie de «l'humain » pour désigner l'espèce humaine) telles que le SIDA, le SRAS, certaines fièvres hémorragiques, voire la peste (Yersina pestis), qui sont dues à un agent pathogène issu de la faune sauvage ayant acquis l'aptitude à se propager dans les groupes humains, entrent pour partie dans le cadre de cette revue; mais celle-ci se limite à l'étude et à la compréhension de leur origine, plus précisément aux conditions de passage de l'agent pathogène d'une population animale sauvage en liberté à l'humain. Ce passage peut avoir lieu avec ou sans l'interposition d'un relais représenté par une ou des populations animales domestiques ou sauvages détenues en captivité. Pour certaines de ces maladies humaines, des arthropodes assurent une transmission efficace du pathogène (ce sont les maladies dites vectorielles, comme la borréliose de Lyme par exemple).

\section{MICRO-ORGANISMES ET PARASITES, LEUR POUVOIR PATHOGENE}

Pour les besoins de clarté de cet article, nous précisons ci-dessous le sens que nous souhaitons adopter pour les termes relatifs aux agents pathogènes.

La dénomination de certains agents capables de causer une maladie dans une espèce, mais pouvant être tolérés, ou symbiotiques dans une autre, n'est pas simple à résumer : le terme «microbe» fait référence à un être biologique n'étant ni une molécule, ni un composé toxique, visible au travers d'une optique grossissante, par opposition à des métazoaires parasites qui sont visibles à la loupe ou à l'œil nu. Le terme de microbe a l'avantage de ne pas sous-entendre un rôle pathogène. Plus récemment, on a pu lui préférer le terme de « micro-organisme ». Les virus ont été inclus assez récemment comme faisant partie des microbes ou micro-organismes.

Lorsque l'on veut désigner tous les agents qui peuvent engendrer un état pathologique chez au moins certains individus d'une espèce, on se réfère à l'expression " agent pathogène " qui regroupe des micro et macro-parasites, mais peut être aussi employée pour des composés inertes. Le terme de parasite est dans ce cas employé en référence à la fonction écologique parasitaire, mais pas au classement taxonomique qui distingue en médecine, les parasites (protozoaires et métazoaires exerçant une action spoliatrice sur leur hôte mais dont la multiplication ne se produit généralement pas dans les organes de cet hôte) des agents infectieux (virus, bactéries, et autres micro-organismes) qui se multiplient dans les organes et tissus de leur hôte.

"Pathogène " est, en français académique, un adjectif. Mais la littérature anglo-américaine a banalisé son emploi comme substantif : un "pathogène " est donc un agent capable de provoquer une maladie. Nous emploierons l'un ou l'autre des termes en fonction du contexte.

Les microbes et les métazoaires parasites ne sont pas tous pathogènes ; l'immense majorité d'entre eux sont indifférents ou sont tolérés sans dommage par l'organisme qui les héberge : ils peuvent être commensaux ou symbiotiques, et la littérature anglaise emploie souvent le vocable de "mutualiste ». Aussi, pour établir un lien de pathogénicité entre un micro-organisme hébergé par la faune et l'humain, c'est-à-dire l'existence d'une zoonose d'origine sauvage, la détection de la maladie chez l'humain est une étape indispensable. La caractéristique commune des agents transmissibles qui font l'objet de cette revue est leur capacité à se transmettre naturellement entre différentes espèces pourvu que les populations réceptives soient en relation. Ces agents "parasites généralistes " ou « multi-hôtes», (termes utilisés dans les publications scientifiques) trouvent dans un éventail d'espèces hôtes les capacités adaptatives permettant d'infecter les hôtes; ils n'ont aucune "barrière d'espèce " à franchir si une voie de transmission permet le passage d'une population d'hôtes d'une espèce donneuse à une espèce réceptrice (Borremans et al., 2019). A cet égard, l'utilisation de la terminologie "barrière d'espèce " ne serait-elle pas de nature à induire quelques biais cognitifs sur la perception des phénomènes d'émergence et de transmission? Elle donne notamment une image caricaturale d'états de mode de transmission inter-espèces classiquement hermétique, démentie par la notion même de zoonose.

\section{LES POPULATIONS ANIMALES « SOURCES " D'AGENTS PATHOGENES TRANSMISSIBLES}

Les animaux sauvages, principalement les mammifères et dans une moindre mesure les oiseaux, hébergent souvent et probablement majoritairement, les microbes reconnus responsables des nouvelles maladies infectieuses ou parasitaires des animaux domestiques et des humains.

Au départ, lorsque sont publiées les premières études sur les maladies et pathogènes émergents, c'est généralement par le terme de "faune sauvage" (wildlife en anglais) qu'ont été désignées les espèces animales sources de certaines contaminations. Le code de l'OIE (Organisation mondiale de la santé animale) définit et distingue : l'animal sauvage, l'animal sauvage en captivité, l'animal domestique « féral » et l'animal domestique proprement-dit (OIE, 2019). Pour sa part, la loi de santé animale de l'Union Européenne, promulguée en 2016, entrée en vigueur en 2020, ne distingue que les animaux " détenus " et les animaux en liberté (EU regulation, 2016). La faune sauvage en liberté se distingue, par son mode de vie, des populations d'animaux domestiques ou sauvages maintenues en captivité (animaux de parcs zoologiques, de réserves de chasse...). En outre, la littérature scientifique abonde d'études sur le rôle des animaux dits "synanthropiques " qui appartiennent à des espèces sauvages et commensales liées durablement à la présence humaine, comme de très nombreuses espèces de chauve-souris et de rongeurs, dans la transmission animal-humain. Enfin, des «espèces relais» (bridge-hosts en anglais) peuvent aussi constituer un lien (nécessaire ou non pour la transmission selon les pathogènes) entre des individus contaminés et des populations animales réservoirs d'agents pathogènes. Ainsi, la transmission du virus Ebola pourrait se faire à partir d'un réservoir qui reste méconnu via des espèces relais favorisant la transmission humaine comme certaines chauves-souris et les grands primates (Leendertz, 2016). L'étude du maintien et de la transmission des 
agents infectieux dans les populations réservoirs reste une tâche difficile pour le monde scientifique. La prévalence et l'incidence dans ces populations est en effet généralement faible, et la diversité spécifique des populations animales impliquées est telle qu'il est souvent difficile d'y détecter et étudier l'agent infectieux.

\section{UN PEU DE SEMANTIQUE: « SANTE ET BIODI- VERSITE ॥, CE QUE REVELENT (OU CACHENT) LES MOTS}

Dans ce paragraphe, nous essayons d'adopter un point de vue inspiré par les sciences humaines et sociales, bien qu'aucun des signataires de ce mémoire ne soit issu de ces disciplines. Un recueil plus détaillé pourra être trouvé dans l'ouvrage de Morand et Lajaunie (2018) qui explore les recherches associant la santé et la biodiversité, l'écologie évolutive des maladies infectieuses et non infectieuses, l'éthique, le droit, et enfin les politiques publiques. Nous avons néanmoins jugé important de relativiser les connaissances acquises par les sciences biologiques en les confrontant à des points de vue issus de la sociologie ou de l'anthropologie. Le néologisme de «biodiversité " est d'apparition récente et sa définition mérite une attention particulière. Le concept remonte au "Sommet de la terre ", conférence de l'Organisation des Nations Unies, tenue à Rio de Janeiro, Brésil, en 1992 ; c'est à dire peu après l'irruption des idées et concepts de maladies émergentes. En écologie (la discipline scientifique) les concepts de "richesse spécifique " et de "diversité biologique " ont précédé la notion de "biodiversité " (Begon et al., 1986). L'étude de la richesse spécifique utilise des indicateurs écologiques, issus de la théorie de l'information permettant l'approche analytique de phénomènes complexes (Shannon et al., 1948). Les indicateurs de richesse spécifique, notamment l'indice de diversité de Shannon, prennent en compte le nombre d'espèces et pour chacune d'elles, le nombre d'individus.

La " biodiversité " a pris un sens particulier dans le cadre des projets de développement "soutenable " (ou durable). Elle constitue une "valeur " éthique, sociétale ou politique dont les écologues français se sont emparés (Barbault, 2005).

On observe notamment dans la littérature de vulgarisation et les médias, un glissement du sens de l'expression " protection de la nature " vers celui de "préservation de la biodiversité ». Ce dernier mot comporte une notion quasi-philosophique de «tissu planétaire " dont l'interprétation prend en compte toutes les relations entre les êtres vivants sans exclure ni accorder une place dominante aux êtres humains.

Puisque l'objectif de notre mémoire est d'examiner l'association entre biodiversité et santé, il convient d'interroger également le concept de santé. Celui-ci semble avoir en effet évolué d'un descripteur de bien-être des populations humaines vers une valeur sociétale, associée à de nombreuses préoccupations humaines, en particulier celle de la biodiversité. La philosophe Sarah Carvallo, professeure de philosophie au laboratoire Logiques de l'Agir (sic) de l'université de Franche-Comté a présenté une conférence pendant que nous préparions ce mémoire (Carvallo, 2020). Il nous a semblé que son point de vue méritait d'être résumé ici : la définition de l'Organisation Mondiale de la Santé (OMS) (1948) intégrait une dimension sociale et psychologique pour concevoir la santé humaine comme
" un état complet de bien-être physique, social et mental ", excluant la dimension environnementale (Morand \& Lajaunie, 2018). Comme nous le mentionnons, les maladies infectieuses émergentes ont suscité l'apparition de nouveaux paradigmes de santé (One Health et autres, voir la suite de ce chapitre) qui associent étroitement santé humaine, animale et environnementale. Des philosophes, chercheurs et praticiens tentent désormais de mettre en place une approche pluridisciplinaire du soin qui devra associer des paramètres à la fois biologiques, sociaux et écologiques.

La perception et la compréhension par le public et les décideurs de certaines études relatives aux liens entre les concepts de biodiversité et de santé, peuvent rendre la liaison plus ou moins convaincante en fonction de l'adhésion de ces personnes aux valeurs associées aux deux concepts. Une adhésion à ces valeurs est nécessaire pour les intégrer dans l'action et les faire entrer dans la pratique. Il y a donc, selon nous, une profonde aspiration de la société à l'existence d'un lien bénéfique entre santé et biodiversité. En particulier via la démarche "One Health " (Karesh et al., 2012) même si l'adhésion à cette approche "One Health » n'est pas unanime. Le concept Ecohealth par exemple, peut se traduire en français de deux façons "santé des écosystèmes " (Rapport et al., 1998; Sandifer et al., 2015) ou écologie de la santé (Guégan et al., 2004; Gauthier-Clerc et al., 2010); cette dernière approche se revendiquant comme une nouvelle discipline scientifique. On peut également citer Global Health (santé mondiale ou santé globale) ou Planetary Health (santé planétaire)... ; pour certains publics et décideurs en santé publique, ces concepts ne semblent convaincants qu'à l'aune des orientations professionnelles des lecteurs ou de la méthodologie des auteurs (Manlove et al., 2016; Cunningham et al., 2017) « Il apparaît donc d'une part une difficulté à véritablement intégrer les trois dimensions de la santé humaine, animale et environnementale, et d'autre part une difficulté à mettre en politique les concepts avancés depuis vingt ans pour proposer un progrès dans ce domaine » (Morand et al., 2020).

De nos jours, les activités humaines ont une incidence majeure (globale) sur les écosystèmes terrestres et aquatiques (Crutzen et al., 2003), nous devons donc aussi interroger les représentations anthropologiques (conscientes ou inconscientes) des conséquences écologiques et sanitaires de cette influence. La réflexion sur le thème de ce mémoire est souvent anthropocentrée, pour ne pas dire anthropocentrique. Nous devons donc nous interroger sur l'emploi de métaphores comme "santé des écosystèmes " (Rapport, 1989) ou encore "santé de la Terre " (Leopold, 2014), voire de biais cognitifs « barrière d'espèce ». Ces représentations orientent la définition des programmes de recherche et en amont, influencent les motivations profondes des financements de ces études scientifiques ; la vision anthropocentrée des sujets liant ou opposant la perte de biodiversité et la santé, influence également l'intégration ou l'ignorance de ces sujets dans l'enseignement universitaire et technologique.

\section{DE LA TRANSMISSION A LA PROPAGATION}

La transmission s'entend ici comme le passage du pathogène d'un individu à un autre, la propagation décrivant la progression de ce pathogène dans une population. 
L'écologie des espèces animales sources des microbes et métazoaires parasites provoquant des maladies émergentes, contribue à expliquer les modalités de passage des agents transmissibles d'intérêt pour nous, de la faune sauvage à l'humain et aux animaux domestiques. On entend par «sympatrie " la coexistence de deux espèces dans la même zone (étendue de surface limitée) ; la "syntopie " est la coexistence de deux espèces dans le même habitat naturel. L'ensemble des êtres vivants (en sympatrie ou syntopie) et leurs interactions (la biocénose) sont plongés dans un ensemble d'éléments physiques ou chimiques du milieu appelé biotope. Ces proximités spatiales facilitent le passage de pathogènes entre deux (ou plusieurs) populations voisines (humains et animaux commensaux par exemple).

Les animaux de production représentent $80 \%$ de la biomasse des vertébrés terrestres, leur concentration et leur faible diversité génétique, ainsi que leur position à l'interface de l'humain et de la faune sauvage, semblent jouer un rôle primordial dans la propagation des pathogènes humains émergents (Rohr et al., 2010; Morand, 2020).

La dégradation des espaces naturels, leur nette réduction en surface (cas des forêts primaires et de la déforestation/fragmentation par exemple) et la modification des pratiques humaines, viennent rompre l'équilibre des écosystèmes et les obstacles physiques et comportementaux entre populations qui occupaient au préalable des aires disjointes et des niches écologiques différentes, rendant possibles des transmissions inter-espèces et l'évolution d'agents pathogènes auparavant limitées à la faune sauvage (Randolph et al., 2010).

Les connaissances disponibles sur la relation écologique entre des populations animales " réservoirs " et des populations d'animaux domestiques et d'humains sont encore lacunaires (Haydon et al., 2002; Viana et al., 2014). La notion de réservoir épidémiologique a considérablement évolué à mesure que s'approfondissaient les connaissances récentes sur les maladies émergentes. On dispose de peu de résultats probants pour documenter les liens entre la persistance de l'agent pathogène dans la population source et sa transmission aux humains. Cette transmission peut se faire dans l'environnement, par l'intervention d'un "véhicule passif " (notamment les animaux domestiques qui peuvent jouer un rôle amplificateur comme c'est le cas pour la transmission des virus Nipah avec le rôle du porc ou Hendra avec le rôle du cheval) ou par un vecteur arthropode, voire même par contact direct.

\section{MALADIES EMERGENTES : " UNE EPIDEMIE D'EPIDEMIES ॥ ?}

Des traces d'épidémies dans les populations humaines sont connues au moins dès le Néolithique. Ces épidémies se sont répandues à l'occasion des déplacements humains à différentes époques préhistoriques et historiques. Selon la conception de N. Gualde (2006), nous sommes entrés dans la quatrième transition épidémique (sic), celle de la globalisation des échanges.

S. Morand (2016) étend les perspectives de compréhension des épidémies en incluant le rôle de l'évolution des relations écologiques que les humains entretiennent avec les autres espèces vivantes. Les interactions entre les humains, les animaux domestiques et sauvages, l'environnement naturel et anthropisé, sont à la source de l'apparition de zoonoses (anciennes et nouvelles) et de maladies infectieuses et parasitaires émergentes. Les épidémies provoquées chez les humains par ces organismes ont augmenté en nombre depuis le XXème siècle (pendant lequel la population humaine a triplé entraînant un accroissement encore plus spectaculaire de la biomasse des animaux de production). Nous sommes passés d'une ou deux pandémies par siècle, à plusieurs par décennie mais la tendance actuelle fait l'objet de controverses car si de nouvelles épidémies apparaissent en grand nombre, leur incidence (en nombre de cas humains) a aussi tendance à diminuer (Smith et al., 2014). Il s'agit en effet du cas de la plupart des maladies infectieuses émergentes des dernières années avant l'émergence de la COVID-19. Les infections à SARS-CoV-1 en 2002 et 2003, et MERS-CoV depuis 2012, à elles deux ont entraîné environ 900 morts ; l'épidémie à virus Ebola en Afrique de l'Ouest (2017-2019) a entraîné, selon diverses sources citant l'OMS, entre 10000 et 20000 morts au total, mais à la même époque le paludisme tuait annuellement plus de 400000 personnes (WHO, 2019).

Des zoonoses, à proprement parler, ont pu en Europe émerger, ré-émerger, ou prendre une nouvelle ampleur économique et sanitaire au cours des soixante dernières années (depuis l'apparition de la rage vulpine en 1968, en France, un rapport de l'ENSV de 2017, a comptabilisé ainsi 27 évènements d'émergence ou de ré-émergence impliquant la faune sauvage en France (Balon et al., 2017)). La source primaire est la faune sauvage autochtone ou, dans une bien moindre mesure, certaines espèces introduites et parfois envahissantes. A titre d'exemple, nous pouvons citer le rôle du Tamia de Sibérie (Eutamias sibiricus) en forêt de Sénart (au sud de l'Île de France) qui contribuerait à développer un foyer de maladie de Lyme dans cette forêt péri-urbaine très fréquentée (Marsot et al., 2013).

Il y a donc bien une problématique importante à prendre en considération, notamment sur le "vieux " continent européen. Néanmoins, les rapports annuels sur la situation des zoonoses en Europe montrent qu'en nombre de cas détéctés, les principales (salmonelloses, campylobactérioses, entérocolites hémorragiques à $E$. coli vérotoxiques et, dans une moindre mesure, les yersinioses) sont d'origine alimentaire et liées à l'élevage (EFSA et al., 2019). Sur ce continent, les zoonoses et maladies vectorielles transmises par la faune sauvage (fièvre hémorragique à syndrome rénal, leptospirose, maladie de Lyme principalement) ne revêtent qu'une importance faible, en nombre de cas annuellement détectés (quelques dizaines de milliers au pire, parmi les 400 millions d'européens). Cependant, ces estimations sont soumises à des biais (sous-estimation du fait de pathogènes entraînant des symptômes non spécifiques et/ou qui ne nécessitent pas d'avis médical ou de notification offcielle) et sont toujours susceptibles d'évoluer. En revanche, les maladies animales portées ou transmises par la faune sauvage prennent une place économique croissante dans l'élevage européen ; la coexistence entre animaux sauvages et animaux d'élevage devient aujourd'hui difficile et constitue une préoccupation sanitaire et économique importante (par exemple : peste porcine africaine, Influenza aviaire hautement pathogène). 
Les « nouvelles » épidémies, voire les pandémies, trouvent essentiellement leur source primaire dans la faune sauvage des zones inter-tropicales de certaines régions d'Asie, d'Afrique ou d'Amérique (Allen et al., 2017; Guégan et al., 2020). Lorsque la maladie infectieuse nouvelle est une zoonose, la transmission interhumaine peut être exceptionnelle, voire inexistante (comme pour l'influenza H5N1). Toutefois, les agents pathogènes responsables de nouvelles pandémies, comme nous l'avons précédemment souligné, sont principalement des micro-organismes et parasites qui se sont adaptés à l'espèce humaine et peuvent se transmettre entre humains sans besoin des animaux (comme pour le SARS-CoV-2) (Haider et al., 2020). L'étude du vivant (la biologie) ne peut être appréciée sans prendre en compte les différentes échelles à travers lesquelles elle se réalise, que ce soit à l'échelle de la cellule, de l'organisme, des populations, des peuplements ou des écosystèmes. Ainsi, l'étude des maladies infectieuses émergentes n'échappe pas à cette observation et l'étude spatio-temporelle de l'évolution des pathogènes, pour qu'elle soit complète et qu'elle permette une vision globale des mécanismes qui la régissent, nécessite une analyse à ces différentes échelles (organisme, population, peuplement) en différents lieux ou habitats (Magnusson et al., 2020), et à différentes échelles de temps (Ezenwa et al., 2015).

Diverses catégories taxinomiques d'agents pathogènes peuvent être cause de zoonoses ou de maladies émergentes; toutefois les virus à $\mathrm{ARN}$, du fait de leur capacité de mutations adaptatives liée à leur mode de réplication, se multipliant dans le cytoplasme, sont significativement plus souvent en cause que d'autres agents pathogènes (Pulliam et al., 2009). A l'opposé, les métazoaires parasites qui évoluent sur un laps de temps bien plus lent apparaissent peu comme agents de maladies infectieuses émergentes (Cleaveland et al., 2001; Wells et al., 2020).

Comme mentionné précédemment, depuis 20, voire 30 ans, de très nombreuses études ont été publiées sur ces relations (Smith et al., 2010). Pourtant, les controverses qui ont agité les médias pendant la crise de la COVID-19 laissent transparaître le manque d'informations ou de prise en compte du risque zoonotique qui était pourtant déjà connu de la communauté scientifique. Par exemple, le programme d'études pluridisciplinaire " PREDICT " de l'USAID (United States Agency for International Development), ("PREDICT program," 2009) avait notamment pointé le marché "vivant " (Wet market) de la ville de Wuhan en Chine comme un point chaud (hot spot) pour le démarrage d'une future épidémie. De surcroit, ce même programme avait pressenti le groupe des coronavirus comme source d'une très plausible future pandémie. Confirmant cette crainte, l'OMS avait mis le SRAS et le MERS sur la liste des maladies prioritaires en terme de recherche et développement afin de signaler que les coronavirus soient un groupe à surveiller avec acuité (WHO, 2018).

Plusieurs projets de recherche de l'UE (en particulier EDEN, EDEN-Next, WildTech, APHAEA, MedVetNet pour ne citer que ceux dont nous avons connaissance) portaient également sur la problématique des maladies infectieuses émergentes en relation avec la faune sauvage.

Dans ce contexte, la crise sanitaire engendrée par la pandémie de COVID-19 suggère qu'une meilleure prise en compte du danger de portage des pathogènes dans l'environnement est nécessaire ; une bonne connaissance de ces dangers et une surveillance épidémiologiques ciblant les expositions à ces dangers doit se mettre en place pour permettre une prévention efficace du risque d'émergence de maladies infectieuses.

\section{LA BIODIVERSITE : UN DANGER OU UNE SECURITE SANITAIRE?}

Un des sujets scientifiques les plus débattus et au cour de la problématique de ce mémoire reste la question de savoir si la diversité biologique des écosystèmes favorise ou au contraire limite le nombre d'émergences de zoonoses et de maladies infectieuses (Keesing et al., 2010 ; Wood et al., 2014 ; Johnson et al., 2015; Rohr et al., 2020). Des milliers de micro-organismes restent à inventorier dans les populations d'animaux sauvages. Quelques centaines de virus sont reconnus comme agents de maladies humaines et des investigations par sondage suggèrent qu'ils représentent moins de $1 \%$ de la diversité des virus hébergés par la faune mammalienne ou aviaire sauvage, le reste étant encore à découvrir (Anthony et al., 2013). Ces virus et autres pathogènes inconnus peuvent, à l'occasion de combinaisons d'événements fortuits, infecter et rendre malades des personnes humaines.

L'émergence des nouvelles maladies transmissibles semble ensuite se produire par étapes successives dont la description dans ses grandes lignes fait consensus chez la plupart des scientifiques, mais revêt une complexité considérable dans le détail de la chaîne de transmission (Figure 2).

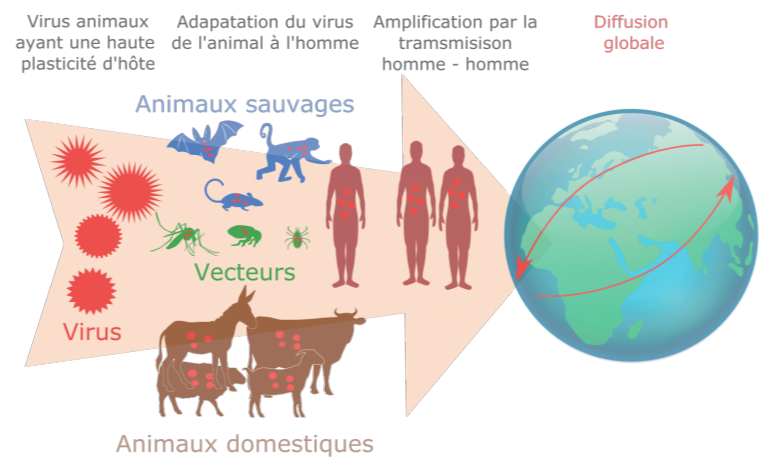

Figure 2 : Propriété pandémique d'un virus zoonotique d'origine animale montrant une transmission de l'animal vers l'humain puis une expansion par transmission inter-humaine (Illustration de Kreuder et al. (2015)).

Les perturbations de l'environnement " naturel " ou déjà modifié (urbanisation, déforestation ou envahissement par des broussailles "bush encroachement ", irrigation, exploitations minières, conflits armés,...) semblent faciliter, voire permettre le passage des pathogènes à des populations humaines confrontées à un habitat naturel en proie à des modifications rapides, voire brutales. Ces modifications incluent des déséquilibres écologiques (alimentation, compétition, prédation, parasitisme...), une modification des communautés en faveur d'espèces dont les traits de vie sont corrélés au statut de réservoir (Gibb et al., 2020), et à un appauvrissement de la diversité spécifique (MacDonald et al., 2019; Guégan et al., 2020; Halliday et al., 2020). 
Certains groupes humains engagés dans des activités au contact du milieu "sauvage " (chasseurs, pêcheurs, forestiers, réfugiés, travailleurs manuels précaires, ...) ou au contact des animaux sauvages eux-mêmes (personnel des marchés de "viande de brousse ", consommateurs, populations de chasseurs-cueilleurs) sont particulièrement exposés à l'infection (Wolfe et al., 2007). Il en va de même pour les éleveurs et leur entourage qui sont particulièrement exposés lorsque l'infection de la faune sauvage est transmise et amplifiée par une espèce domestique ("grippe " aviaire à virus influenza $\mathrm{H} 5 \mathrm{~N} 1$, infection à virus Nipah ou Hendra où les animaux domestiques ont servi d'espèces relais entre le réservoir et l'humain) (Gibb et al., 2020).

Depuis une vingtaine d'années, des recherches étayent l'hypothèse qu'un « effet de dilution » réduit la probabilité de zoonoses dans les écosystèmes à forte diversité spécifique, mais là encore dans des conditions souvent particulières (Ostfeld et al., 2000; Magnusson et al., 2020; Rohr et al., 2020). Le rôle des espèces réservoirs dans le phénomène de dilution apparaît plus important que celui des espèces vectrices qui tendent, au contraire, pour ce type de transmission à amplifier le processus infectieux (Figure 3); ce constat montre l'importante hétérogénéité spatio-temporelle qui peut exister entre effet de dilution et effet d'amplification qui peuvent intervenir et coexister (Roche et al., 2011). Au moment où ce mémoire est rédigé, le sujet continue d'alimenter la recherche sur la complexité des phénomènes de persistance et de propagation des pathogènes.

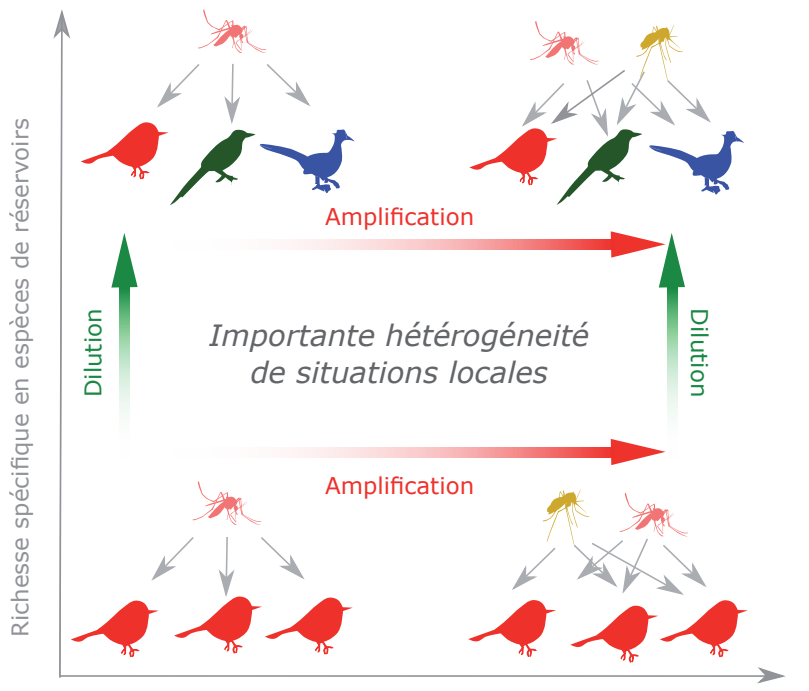

Richesse spécifique en espèces de vecteurs

Figure 3 : Représentation schématique de l'influence de la richesse spécifique des écosystèmes et du rôle épidémiologique associé des espèces sur les phénomènes de dilution et d'amplification des pathogènes (Illustration adaptée de Roche et Guégan, 2011).

\section{CONCLUSION : MIEUX COMPRENDRE POUR MIEUX ANTICIPER, MIEUX ENSEIGNER ET INFORMER POUR MIEUX PREVENIR}

Le SARS-CoV-2, virus responsable de la pandémie de la COVID-19 a déstabilisé le fonctionnement de nos sociétés à l'échelle planétaire. Cette crise mondiale a soulevé de nombreux débats relatifs au caractère zoonotique du virus, très probablement originaire de la faune sauvage mais adapté à une transmission interhumaine, dans des conditions qui ne sont pas encore éclaircies au moment où cette note est rédigée. Les épidémies ont toujours existé mais l'augmentation de leur fréquence ces dernières décennies semble confirmer que le monde actuel tend à favoriser tant leur émergence que leur propagation.

L'existence d'un lien entre maladies émergentes et biodiversité a fait l'objet de nombreuses publications et rapports d'experts. En fonction des contextes, cette corrélation semble être tantôt négative (favorable aux pathogènes et à leur transmission) ou tantôt positive (favorable à la santé en réduisant la transmission). Toutefois une érosion de la biodiversité favorisant une augmentation des émergences est plus largement documentée : les épidémies d'origine zoonotique sont positivement corrélées à une perte de biodiversité et pourraient renforcer cette perte de biodiversité. En effet, les espèces sauvages les plus spécialisées, très adaptées à l'exploitation du milieu naturel dans lequel elles vivent, hébergent des pathogènes eux-mêmes spécialisés, moins enclins à se propager à d'autres espèces. Ces espèces, très dépendantes de leur écosystème, sont les plus menacées quand le milieu naturel est modifié. Dans ce même milieu, les espèces généralistes sont favorisées par l'altération de l'écosystème et peuvent se développer; les pathogènes qu'elles hébergent sont aptes à coloniser de nouveaux hôtes et par conséquent peuvent passer aux animaux domestiques et à l'humain (Morand \& Figuié, 2018).

La perturbation des biotopes dans lesquels nous vivons, par la modification des habitats et leur exploitation croissante par les humains, crée en effet des opportunités de transmission de pathogènes aux humains et à leurs animaux domestiques. La faune sauvage peut héberger une grande diversité de micro-organismes et a fortiori donc de pathogènes pour l'humain résultant de la diversité zoologique des hôtes. Elle est donc une source potentielle de nouveaux pathogènes pour l'espèce humaine à l'occasion de nouveaux contacts (destructions de biotopes) mais elle est également partie prenante $\mathrm{du}$ fonctionnement des écosystèmes dans lesquels nous évoluons et dont nous dépendons. Une meilleure compréhension de l'impact de la biodiversité sur notre sécurité sanitaire et une meilleure information et éducation sur notamment les risques de l'exploitation, voire la sur-exploitation, actuelle qui est faite des écosystèmes «naturels» et anthropisés, permettra de mieux comprendre les mécanismes d'émergence et de réémergence. Afin de prévenir l'établissement de nouvelles maladies, l'étude pluridisciplinaire de ces phénomènes paraît plus que jamais essentielle.

Les pathogènes participent pleinement aux relations entre organismes et populations interagissant dans les écosystèmes. Considérer l'origine et la propagation des pandémies du point de vue de l'écologie des populations et des écosystèmes, peut aider à mieux comprendre leurs causes profondes et à concevoir des solutions pour prévenir leur émergence. Une meilleure intégration de cette approche (écologie de la santé) par une recherche inter- et trans-disciplinaire impose que les chercheurs travaillant sur l'écologie des pathogènes et l'environnement contribuent aux stratégies de prévention et de contrôle des pandémies, en collaboration avec les autres professionnels de la santé humaine et animale, et avec l'appui des sciences humaines. 


\section{REMERCIEMENTS}

Les auteurs remercient l'Académie Vétérinaire de France, en particulier les membres du Groupe de réflexion sur la biodiversité, pour leur avoir offert l'opportunité de rédiger ce mémoire et leur appui dans le travail préparatoire de rédaction.

Les auteurs tiennent également à remercier les relecteurs anonymes qui ont permis de préciser les propos et enrichir significativement ce manuscrit.

\section{BIBLIOGRAPHIE}

- Abbate, J. L., Galan, M., Razzauti, M., Sironen, T., Voutilainen, L., Henttonen, H., Gasqui, P., Cosson, J.-F., Charbonnel, N. (2020). Pathogen community composition and co-infection patterns in a wild community of rodents. bioRxiv, doi:10.1101/2020.02.09.940494

- Abdallat, M. M., Abroug, F., Al Dhahry, S. H. S., Alhajri, M. M., Al-Hakeem, R., Al Hosani, F. I., Al Qasrawi, S. M. A., Al-Romaihi, H. E., Assiri, A., Baillie, J. K., et al. (2013). State of knowledge and data gaps of middle east respiratory syndrome coronavirus (MERS-CoV) in humans. PLoS Curents Outbreaks, 5

DOI: $10.1371 /$ currents.outbreaks.0bf719e352 e7478f8ad85fa30127ddb8.

- Alexander, D. J. (2007). Summary of avian influenza activity in Europe, Asia, Africa, and Australasia, 2002-2006. Avian Diseases, 51(SU PPL. 1), 161-166. doi:10.1637/7602-041306r.1

- Allen, T., Murray, K. A., Zambrana-Torrelio, C., Morse, S. S., Rondinini, C., Di Marco, M., Breit, N., Olival, K. J., Daszak, P. (2017). Global hotspots and correlates of emerging zoonotic diseases. Nature Communications, 8(1). doi:10.1038/s41467-017-00923-8

- Anthony, S. J., Epstein, J. H., Murray, K. A., Navarrete-Macias, I., Zambrana-Torrelio, C. M., Solovyov, A., Ojeda-Flores, R., Arrigo, N. C., Islam, A., Khan, S. A., et al. (2013). A strategy to estimate unknown viral diversity in mammals. mBio, 4(5).

doi: $10.1128 / \mathrm{mBio}$. 00598-13

- Ashford, E. (2003). Individual Responsibility And Global Consequences. Philosophical Books, 44(2), 100-110. doi:https://doi.org/ 10.1111/1468-0149.00291_2

- Balon, C., Revollal, B. (2017). Crises sanitaires impliquant la faune sauvage. Marcy L'Etoile, France: http://www.ensv.fr/wp-content/uploads/2017/10/GEST-ENSV-rapp ort-FINAL.pdf

- Barbault, R. (2005). Biodiversité, écologie et sociétés. Écologie \& politique, 30(1), 27-40.

- Begon, M., Harper, J. L., Townsend, C. R. (1986). Ecology: individuals, populations and communities.

- Berger, A., Drosten, C., Doerr, H. W., Stürmer, M., Preiser, W. (2004). Severe acute respiratory syndrome (SARS) - Paradigm of an emerging viral infection. Journal of Clinical Virology, 29(1), 13-22.

doi:10.1016/j.jcv.2003.09.011

- Borenstein, M., Hedges, L. V., Higgins, J. P. T., Rothstein, H. R. (2009). Introduction to Meta-Ana Ed. Wiley.

- Borremans, B., Faust, C., Manlove, K. R., Sokolow, S. H., Lloyd-Smith, J. O. (2019). Cross-species pathogen spillover across ecosystem boundaries: Mechanisms and theory. Philosophical Transactions of the Royal Society B: Biological Sciences, 374(1782). doi:10.1098/rstb.2018.0344

- Calisher, C. H., Childs, J. E., Field, H. E. Holmes, K. V., Schountz, T. (2006). Bats: Important reservoir hosts of emerging viruses. Clinical Microbiology Reviews, 19(3), 531-545. doi:10.1128/CMR.00017-06

- Carvallo, S. (Producer). (2020, 7 juilllet 2020). Quel concept de santé a été mobilisé par et dans la pandémie? Penser la pandémie. Retrieved from https://actu.univ-fcomte.fr/agenda/ quel-concept-de-sante-ete-mobilise-par-et-da ns-la-pandemie\#.XrklQsDgqUk

- Cleaveland, S., Laurenson, M. K., Taylor, L. H. (2001). Diseases of humans and their domestic mammals: Pathogen characteristics, host range and the risk of emergence. Philosophical Transactions of the Royal Society B: Biological Sciences, 356 (1411), 991-999. doi:10.1098/rstb.2001.0889

- Crutzen, P. J., Steffen, W. (2003). How long have we been in the Anthropocene era? An Editorial Comment. Climatic Change, 61(3), 251-257. doi:10.1023/B:CLIM.0000004708.74871.62

- Cunningham, A. A., Daszak, P., Wood, J. L. N. (2017). One health, emerging infectious diseases and wildlife: Two decades of progress? Philosophical Transactions of the Royal Society B: Biological Sciences, 372(1725). doi: 10.1098/rstb.2016.0167

- Daszak, P., Olival, K. J., Li, H. (2020). A strategy to prevent future epidemics similar to the 2019-nCoV outbreak. Biosafety and Health, 2(1), 6-8. doi:https://doi.org/10.1016/j. bsheal.2020.01.003

- EFSA, ECDC. (2019). The European Union One Health 2018 Zoonoses Report. https://efsa.onlinelibrary.wile y.com /doi/10. 2903/j.efsa.2019.5926

- REGULATION (EU) 2016/429 of The European Parliament And of The Council, OJ L 84, 31.3.2016 C.F.R. (2016).

- Ezenwa, V. O., Prieur-Richard, A. H., Roche, B., Bailly, X., Becquart, P., García-Peña, G. E., Hosseini, P. R., Keesing, F., Rizzoli, A., Suzán, G., et al. (2015). Interdisciplinarity and Infectious Diseases: An Ebola Case Study. PLoS Pathogens, 11(8).

doi:10.1371/journal.ppat.1004992

- Gauthier-Clerc, M., Thomas, F. (2010). Ecologie de la santé et Biodiversité. Bruxelles, Belgium: De Boeck.

- Gibb, R., Redding, D. W., Chin, K. Q. Donnelly, C. A., Blackburn, T. M., Newbold, T., Jones, K. E. (2020). Zoonotic host diversity increases in human-dominated ecosystems. Nature, 584(7821), 398-402. doi: $10.1038 / \mathrm{s} 41586-020-2562-8$

- Gualde, N. (2006). Comprendre les épidémies:
La coévolution des microbes et des hommes (Les empêcheurs de penser rond Ed.).

- Guégan, J. F., Ayouba, A., Cappelle, J., de Thoisy, B. (2020). Forests and emerging infectious diseases: Unleashing the beast within. Environmental Research Letters, 15(8). doi: 10.1088/1748-9326/ab8dd7

- Guégan, J. F., Renaud, F. (2004). Biodiversité et changements globaux. I Vers un Ecologie de la Santé ! (pp. 100-135). Paris (France): ADPF Ministère des Affaires Etrangères.

- Haider, N., Rothman-Ostrow, P., Osman, A. Y., Arruda, L. B., Macfarlane-Berry, L., Elton, L., Thomason, M. J., Yeboah-Manu, D. Ansumana, R., Kapata, N., et al. (2020). COVID-19-Zoonosis or Emerging Infectious Disease? Frontiers in Public Health, 8. doi: 10.3389/fpubh.2020.596944

- Halliday, F. W., Rohr, J. R., Laine, A. L. (2020). Biodiversity loss underlies the dilution effect of biodiversity. Ecology Letters. doi: $10.1111 /$ ele. 13590

- Haydon, D. T., Cleaveland, S., Taylor, L. H., Laurenson, M. K. (2002). Identifying reservoirs of infection: A conceptual and practical challenge. Emerging Infectious Diseases, 8(12), 1468-1473. doi:10.3201/eid0812.010317

- Hayman, D. T. S. (2019). Bat tolerance to viral infections. Nature Microbiology, 4(5), 728-729. doi:10.1038/s41564-019-0430-9

- Huang, C., Wang, Y., Li, X., Ren, L., Zhao, J., Hu, Y., Zhang, L., Fan, G., Xu, J., Gu, X., et al. (2020). Clinical features of patients infected with 2019 novel coronavirus in Wuhan, China. The Lancet, 395(10223), 497-506. doi:10.1016/S0140-6736(20)30183-5

- Johnson, C. K., Hitchens, P. L., Pandit, P. S., Rushmore, J., Evans, T. S., Young, C. C. W., Doyle, M. M. (2020). Global shifts in mammalian population trends reveal key predictors of virus spillover risk. Proceedings of the Royal Society B: Biological Sciences, 287(1924). doi: $10.1098 / \mathrm{rspb} .2019 .2736$

- Johnson, P. T. J., Ostfeld, R. S., Keesing, F. (2015). Frontiers in research on biodiversity and disease. Ecology Letters, 18(10), 1119-1133. doi:10.1111/ele.12479

- Jones, K. E., Patel, N. G., Levy, M. A., Storeygard, A., Balk, D., Gittleman, J. L., Daszak, P. (2008). Global trends in emerging infectious diseases. Nature, 451(7181), 990-993. doi:10.1038/nature06536

- Karesh, W. B., Dobson, A., Lloyd-Smith, J. O., Lubroth, J., Dixon, M. A., Bennett, M., Aldrich, S., Harrington, T., Formenty, P., Loh, E. H., et al. (2012). Ecology of zoonoses: Natural and unnatural histories. The Lancet, 380(9857), 1936-1945. doi:10.1016/S0140-6736(12)61678-X 
- Keck, F. (2020). Les Sentinelles des pandémies: Chasseurs de virus et observateurs d'oiseaux aux frontières de la Chine (Z. Sensibles Ed.).

- Keesing, F., Belden, L. K., Daszak, P., Dobson, A., Harvell, C. D., Holt, R. D., Hudson, P. Jolles, A., Jones, K. E., Mitchell, C. E., et al. (2010). Impacts of biodiversity on the emergence and transmission of infectious diseases. Nature, 468(7324), 647-652. doi:10.1038/nature09575

- Kreuder Johnson, C., Hitchens, P. L., Smiley Evans, T., Goldstein, T., Thomas, K. Clements, A., Joly, D. O., Wolfe, N. D., Daszak, P., Karesh, W. B., et al. (2015) Spillover and pandemic properties of zoonotic viruses with high host plasticity. Scientific Reports, 5. doi: 10.1038/srep 14830

- Leendertz, S. A. J. (2016). Testing new hypotheses regarding ebolavirus reservoirs. Viruses, 8(2). doi:10.3390/v8020030

- Leopold, A. (2014). Pour la santé de la terre (D. Corti Ed.).

- Liu, P., Jiang, J. Z., Wan, X. F., Hua, Y., Li, L., Zhou, J., Wang, X., Hou, F., Chen, J., Zou, J., et al. (2020). Are pangolins the intermediate host of the 2019 novel coronavirus (SARS-CoV-2)? PLoS Pathogens, 16(5) doi:10.1371/journal.ppat.1008421

- Luis, A. D., Hayman, D. T. S., O'Shea, T. J., Cryan, P. M., Gilbert, A. T., Pulliam, J. R. C., Mills, J. N., Timonin, M. E., Willis, C. K. R., Cunningham, A. A., et al. (2013). A comparison of bats and rodents as reservoirs of zoonotic viruses: Are bats special? Proceedings of the Royal Society B: Biological Sciences, 280(1756). doi:10.1098/rspb.2012.2753

- MacDonald, A. J., Mordecai, E. A. (2019). Amazon deforestation drives malaria transmis sion, and malaria burden reduces forest clearing. Proceedings of the National Academy of Sciences of the United States of America, 116(44) 22212-22218. doi: 10.1073/pnas. 1905315116

- Magnusson, M., Fischhoff, I. R., Ecke, F., Hörnfeldt, B., Ostfeld, R. S. (2020). Effect of spatial scale and latitude on diversity-disease relationships. Ecology, 101(3) doi: $10.1002 /$ ecy.2955

- Manlove, K. R., Walker, J. G., Craft, M. E., Huyvaert, K. P., Joseph, M. B., Miller, R. S. Nol, P., Patyk, K. A., O’Brien, D., Walsh, D. P., et al. (2016). "One Health" or Three? Publication Silos Among the One Health Disciplines. PLoS Biology, 14(4) doi:10.1371/journal.pbio. 1002448

- Marsot, M., Chapuis, J. L., Gasqui, P., Dozières, A., Masséglia, S., Pisanu, B., Ferquel, E., Vourc'h, G. (2013). Introduced Siberian Chipmunks (Tamias sibiricus barberi) Contribute More to Lyme Borreliosis Risk than Native Reservoir Rodents. PLoS ONE, 8(1). doi:10.1371/journal.pone.0055377

- Morand, S. (2016). La prochaine peste: une histoire globale des maladies infectieuses.

- Morand, S. (2020). Emerging diseases, livestock expansion and biodiversity loss are positively related at global scale. Biological Conservation, 248. doi:10.1016/j.biocon.2020.108707

- Morand, S., Figuié, M. (2018). Emergence of infectious diseases: Risks and Issues fo societies. Matière à Débattre. https://www.qu ae-open.com/produit/94/9782759227723 /emergence-of-infectious-diseases

- Morand, S., Guégan, J. F., Laurans, Y. (2020). De One Health à Ecohealth, cartographie du chantier inachevé de l'intégration des santés humaine, animale et environnementale. IDDRI, Décryptage $\mathrm{N}^{\circ} 04 / 20,4$.

- Morand, S., Lajaunie, C. (2018). Biodiversité et santé: Les liens entre le vivant, le écosystèmes et les sociétés (ISTE éditions ed.).

- Morse, S. S., Mazet, J. A. K., Woolhouse, M. Parrish, C. R., Carroll, D., Karesh, W. B., Zambrana-Torrelio, C., Lipkin, W. I., Daszak, P. (2012). Prediction and prevention of the next pandemic zoonosis. The Lancet, 380(9857), 1956-1965.

doi:10.1016/S0140-6736(12)61684-5

- Murray, K. A., Olivero, J., Roche, B., Tiedt, S., Guégan, J. F. (2018). Pathogeography: leveraging the biogeography of human infectious diseases for global health management. Ecography, 41(9), 1411-1427. doi:10.1111/ecog.03625

- Nava, G. M., Attene-Ramos, M. S., Ang, J. K. Escorcia, M. (2009). Origins of the new influenza $\mathrm{A}(\mathrm{H} 1 \mathrm{~N} 1)$ virus: time to take action. Euro surveillance : bulletin européen sur les maladies transmissibles doi:10.2807/ese.14.22.19228-en

- OIE. (2019). OIE Terrestrial Animal Health Code.

- Olival, K. J., Hosseini, P. R., Zambrana-Torrelio, C., Ross, N., Bogich, T. L., Daszak, P. (2017). Host and viral traits predict zoonotic spillover from mammals. Nature, 546(7660), 646-650. doi:10.1038/nature22975

- Ostfeld, R. S., Keesing, F. (2000). Biodiversity and disease risk: The case of Lyme disease. Conservation Biology, 14(3), 722-728. doi:10.1046/j.1523-1739.2000.99014.x

PREDICT program. (2009). Retrieved from https://ohi.vetmed.ucdavis.edu/programs-pr ojects/predict-project

- Pulliam, J. R. C., Dushoff, J. (2009). Ability to replicate in the cytoplasm predicts zoonotic transmission of livestock viruses. Journal of Infectious Diseases, 199(4), 565-568. doi: $10.1086 / 596510$

- Randolph, S. E., Rogers, D. J. (2010). The arrival, establishment and spread of exotic diseases: Patterns and predictions. Nature Reviews Microbiology, 8(5), 361-371. doi: $10.1038 /$ nrmicro2336

- Rapport, D. J. (1989). What constitutes ecosystem health? Perspectives in Biology and Medicine, 33(1), 120-132. doi: $10.1353 /$ pbm.1990.0004

- Rapport, D. J., Costanza, R., McMichael, A. J. (1998). Assessing ecosystem health. Trends in Ecology and Evolution, 13(10), 397-402. doi:10.1016/S0169-5347(98)01449-9

- Roche, B., Guégan, J. F. (2011). Ecosystem dynamics, biological diversity and emerging infectious diseases. Comptes Rendus Biologies, 334(5-6), 385-392.

doi:10.1016/j.crv i.2011.02.008

- Rohr, J. R., Civitello, D. J., Halliday, F. W. Hudson, P. J., Lafferty, K. D., Wood, C. L.,
Mordecai, E. A. (2020). Towards common ground in the biodiversity-disease debate. Nature Ecology and Evolution, 4(1), 24-33. doi:10.1038/s41559-019-1060-6

- Rohr, J. R., Raffel, T. R. (2010). Linking global climate and temperature variability to widespread amphibian declines putatively caused by disease. Proceedings of the National Academy of Sciences of the United States of America, 107(18), 8269-8274. doi:10.1073/pnas.0912883107

- Salata, C., Calistri, A., Parolin, C., Palù, G. (2020). Coronaviruses: A paradigm of new emerging zoonotic diseases. Pathogens and Disease, 77(9). doi:10.1093/femspd/ftaa006

- Salkeld, D. J., Padgett, K. A., Jones, J. H. (2013). A meta-analysis suggesting that the relationship between biodiversity and risk of zoonotic pathogen transmission is idiosyncratic. Ecology Letters, 16(5), 679-686. doi:10.1111/ele.12101

- Sandifer, P. A., Sutton-Grier, A. E., Ward, B. P. (2015). Exploring connections among nature, biodiversity, ecosystem services, and human health and well-being : Opportunities to enhance health and biodiversity conservation. Ecosystem Services, 12, 1-15. doi:10.1016/j.ecoser.2014.12.007

- Shannon, C. E., Weaver, W. (1948). The Mathematical Theory of Communication. Recent contributions to the mathematical theory of communication. Illinois: University of Illinois Press.

-Smith, K. F., Goldberg, M., Rosenthal, S., Carlson, L., Chen, J., Chen, C., Ramachandran, S. (2014). Global rise in human infectious disease outbreaks. Journal of the - Royal Society Interface, 11(101). doi:10.1098/rsif.2014.0950

- Smith, K. F., Guégan, J. F. (2010) Changing geographic distributions of human pathogens. In: Vol. 41. Annual Review of Ecology, Evolution, and Systematics (pp. 231-250).

- Taylor, L. H., Latham, S. M., Woolhouse, M. E. J. (2001). Risk factors for human disease emergence. Philosophical Transactions of the Royal Society B: Biological Sciences, 356(1411), 983-989. doi: 10.1098/rstb.2001.0888

- Viana, M., Mancy, R., Biek, R., Cleaveland, S., Cross, P. C., Lloyd-Smith, J. O., Haydon, D. T. (2014). Assembling evidence for identifying reservoirs of infection. Trends in Ecology and Evolution, 29(5), 270-279. doi:10.1016/j.tree.2014.03.002

- Wardeh, M., Risley, C., McIntyre, M. K., Setzkorn, C., Baylis, M. (2015). Database of host-pathogen and related species interactions, and their global distribution. Scientific Data, 2. doi:10.1038/sdata.2015.49

- Wells, K., Morand, S., Wardeh, M., Baylis, M. (2020). Distinct spread of DNA and RNA viruses among mammals amid prominent role of domestic species. Global Ecology and Biogeography, 29(3), 470-481. doi:10.1111/geb.13045

- WHO. (2018). Prioritizing diseases for research and development in emergency contexts. Retrieved from https://www.who.int/activ ities/prioritizing-diseases-for-research-anddevelopment-in-emergency-contexts 
- WHO. (2019). World Malaria Report. Retrieved from https://www.who.int/public ations/i/item/9789241565721

- Wolfe, N. D., Dunavan, C. P., Diamond, J. (2007). Origins of major human infectious diseases. Nature, 447(7142), 279-283. doi: $10.1038 /$ nature05775

- Wood, C. L., Lafferty, K. D., DeLeo, G. Young, H. S., Hudson, P. J., Kuris, A. M. (2014). Does biodiversity protect humans against infectious disease? Ecology, 95(4), 817-832. doi: 10.1890/13-1041.1

- Woolhouse, M. E. J., Gowtage-Sequeria, S. (2005). Host range and emerging and reemerging pathogens. Emerging Infectious Diseases, 11(12), 1842-1847. doi:10.3201/eid1112.050997

- Wu, F., Zhao, S., Yu, B., Chen, Y. M., Wang, W., Song, Z. G., Hu, Y., Tao, Z. W., Tian, J. H., Pei, Y. Y., et al. (2020). A new coronavirus associated with human respiratory disease in China. Nature, 579(7798), 265-269. doi:10.1038/s41586-020-2008-3

- Xiao, K., Zhai, J., Feng, Y., Zhou, N., Zhang, X., Zou, J. J., Li, N., Guo, Y., Li, X., Shen, X., et al. (2020). Isolation of SARS-CoV-2-related coronavirus from Malayan pangolins. Nature, 583(7815), 286-289. doi:10.1038/s41586-020-2313-x

- Young, H. S., Wood, C. L., Marm Kilpatrick,
A., Lafferty, K. D., Nunn, C. L., Vincent, J. R. (2017). Conservation, biodiversity and infectious disease: Scientific evidence and policy implications. Philosophical Transactions of the Royal Society B: Biological Sciences, 372(1722). doi:10.1098/rstb.2016.0124

- Zhou, P., Yang, X. L., Wang, X. G., Hu, B., Zhang, L., Zhang, W., Si, H. R., Zhu, Y., Li, B., Huang, C. L., et al. (2020). A pneumonia outbreak associated with a new coronavirus of probable bat origin. Nature, 579(7798), 270-273. doi: 10.1038/s41586-020-2012-7

- Zylberman, P. (2013). Tempête microbienne. Paris: Gallimard. 